\section{Investigación Experimental o Metaanalítica}

Pensar en Movimiento:

Revista de Ciencias del Ejercicio y la Salud ISSN 1659-4436

Vol. 18, No.2, pp. 1 - 18

Abre $1^{\circ}$ de julio, cierra 31 de diciembre, 2020

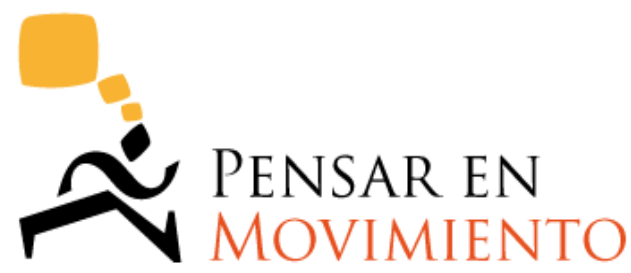

REVISTA DE CIENCIAS DEL EJERCICIO Y LA SALUD

\title{
COMPARACIÓN DEL EFECTO DEL TIPO DE DESCANSO AL REALIZAR EJERCICIO CONTRA RESISTENCIA SOBRE LA PRESIÓN ARTERIAL DE HOMBRES NORMOTENSOS
}

\section{EFFECT OF THE TYPE OF REST ON BLOOD PRESSURE OF NORMOTENSIVE MALES UNDERGOING RESISTANCE EXERCISE}

\section{COMPARAÇÃO DO EFEITO DO TIPO DE DESCANSO AO REALIZAR EXERCÍCIO CONTRARRESISTÊNCIA SOBRE A PRESSÃO ARTERIAL DE HOMENS NORMOTENSOS}

Elizabeth Carpio Rivera, Ph.D (D) 1(A, B, C, D, E), Robelius De Bortoli, Ph.D (D) 2(B, D, E), Andrea Solera Herrera, Ph.D (D) ${ }^{1(B, D, E)}$ y Antônio Cesar Cabral de Oliveira, Ph.D (D) ${ }^{2(B, D, E)}$ elizabeth.carpiorivera@ucr.ac.cr; robelius@yahoo.com.br; andrea.solera@ucr.ac.cr; cabral@infonet.com.br

${ }^{1}$ Escuela de Educación Física y Deportes, Universidad de Costa Rica, San José, Costa Rica

${ }^{2}$ Universidad Federal de Sergipe, São Cristóvão, Brasil

Envío original: 2019-10-01 Reenviado: 2020-08-31

Aceptado: 2020-10-13 Publicado: 2020-12-03

Doi: https://doi.org/10.15517/pensarmov.v18i2.39181

Elizabeth Carpio Rivera y Andrea Solera Herrera son editoras asociadas de la revista. Por esa razón, el manuscrito se maneja en total independencia de ellas hasta que sea rechazado o aprobado. 


\section{RESUMEN}

Carpio Rivera, E., De Bortoli, R., Solera Herrera, A. y Cabral de Oliveira, A.C. (2020). Comparación del efecto del tipo de descanso al realizar ejercicio contra resistencia sobre la presión arterial de hombres normotensos. PensAR en Movimiento: Revista de Ciencias del Ejercicio y la Salud, 18(2), 1-18. Actualmente, se recomienda el ejercicio contra resistencia (ECR) como una de las estrategias no farmacológicas para el control y tratamiento de la presión arterial (PA). Sin embargo, es necesario profundizar esta línea de investigación. El propósito del estudio fue comparar el efecto del tipo de descanso al realizar ECR sobre la PA de participantes normotensos. Participaron nueve hombres; siguiendo un diseño de investigación de medidas repetidas, realizaron tres condiciones experimentales en orden aleatorio: a) Descanso Activo (DA), b) Descanso Pasivo (DP), c) Sesión Control. En las 3 sesiones, se midió la PA y la frecuencia cardíaca (FC) pre-tratamiento, 1 minuto post-sesión y cada 10 minutos posttratamiento durante 120 minutos. Las sesiones experimentales estuvieron precedidas por: mediciones antropométricas, familiarización y aplicación del test de 1RM. En el análisis estadístico se aplicó ANOVA de 2 vías para medidas repetidas, análisis de efectos simples y post hoc de Tukey. Entre los principales resultados, se encontró que, en comparación con la sesión control, los valores de PA disminuyen de forma significativa $(p<.05)$ : 1) al realizar ECR utilizando DP y DA a los 50, 100, 110 minutos post-ejercicio; 2) posterior a ejecutar ECR utilizando DA a los 20, 30, 70, 80,100, 120 minutos; 3) después de finalizada la sesión ECR con DP a los 10, 40 y 120 minutos. Respecto a la medición pre-test, los valores de PA disminuyeron de forma significativa $(p<.05)$ a los $10,20,40,50,60,100$ y 120 minutos post ECR utilizando DP. En conclusión, al comparar con los valores de PA mostrados en un día de control, la ejecución de ECR aplicando DP y DA es funcional en el control de la PA de hombres jóvenes normotensos. En relación al pre-test, se podría indicar que el DP es el que promueve el efecto hipotensivo del ECR.

Palabras clave: descanso activo, descanso pasivo, efecto hipotensor, ejercicio contra resistencia.

\footnotetext{
ABSTRACT

Carpio Rivera, E., De Bortoli, R., Solera Herrera, A. \& Cabral de Oliveira, A.C. (2020). Effect of the type of rest on blood pressure of normotensive males undergoing resistance exercise. PENSAR En Movimiento: Revista de Ciencias del Ejercicio y la Salud, 18(2), 1-18. Resistance exercise is currently recommended as a non-pharmacological strategy to control and treat blood pressure, but further research is necessary concerning this topic. The purpose of this paper is to compare the effect of types of rest on the blood pressure of normotensive individuals while performing resistance exercise. Following a random methodological design with repeated measurements, nine men performed three experimental conditions on different days: a) Active Rest, b) Passive $-2-$

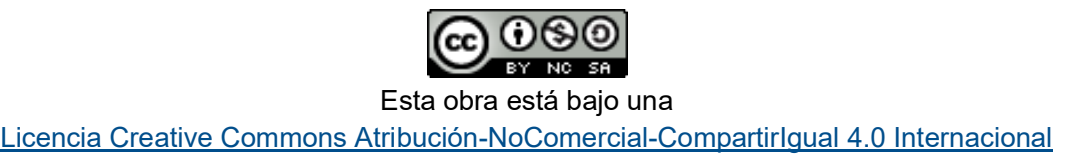


Rest, and c) Control Session. During the three sessions, blood pressure and heart rate measurements were taken pre-treatment, one-minute post-session, and every 10 minutes posttreatment for 120 minutes. Experimental sessions were preceded by anthropometric measurements, familiarization, and taking the 1RM test. The statistical analysis was conducted using two-way ANOVA with repeated measurements and Tukey post-hoc analysis. One of the main results indicated that, compared to the control session, blood pressure decreased significantly $(p<0.05)$ : 1 ) When performing resistance exercise with active and passive rest at 50 , 100, 110 minutes post-exercise; 2) After executing resistance exercise with active rest at 20, 30, $70,80,100$, and 120 minutes; 3) After performing resistance exercise session with passive rest at 10,40 , and 120 minutes. With respect to pre-test levels, blood pressure decreased significantly $(p<0.05)$ at $10,20,40,50,60,100$, and 120 minutes after performing resistance exercise with passive rest. In conclusion, compared with a control day, in the case of young normotensive males, the inclusion of both passive and active rest is functional for controlling blood pressure. In relation to the pre-test, passive rest promotes a hypotensive effect in the study population.

Keywords: active rest, passive rest, hypotensive effect, resistance exercise.

\section{RESUMO}

Carpio Rivera, E., De Bortoli, R., Solera Herrera, A. e Cabral de Oliveira, A.C. (2020). Comparação do efeito do tipo de descanso ao realizar exercício contrarresistência sobre a pressão arterial de homens normotensos. PensAR en Movimiento: Revista de Ciencias del Ejercicio y la Salud, 18(2), 1-18. Recomenda-se, atualmente, o exercício contrarresistência $(E C R)$ como uma das estratégias não farmacológicas para o controle e tratamento da pressão arterial (PA). Não obstante, é preciso aprofundamento nesta linha de pesquisa. O propósito do estudo foi comparar o efeito do tipo de descanso ao realizar ECR sobre a PA de participantes normotensos. Participaram nove homens; seguindo um desenho de pesquisa de medidas repetidas, realizaram três condições experimentais em ordem aleatória: a) Descanso Ativo (DA), b) Descanso Passivo (DP) e c) Sessão Controle. Nas 3 sessões, foram solicitadas a PA e a frequência cardíaca (FC) pré-tratamento, 1 minuto pós-sessão e a cada 10 minutos póstratamento durante 120 minutos. As sessões experimentais foram precedidas por: medições antropométricas, familiarização e aplicação do teste de 1RM. Na análise estatística aplicou-se a ANOVA de 2 vias com medidas repetidas, a análise de efeitos simples e o teste pós-hoc de Tukey. Entre os principais resultados, observou-se que, em comparação com a sessão controle, os valores de PA diminuíram de maneira significativa $(p<0,05)$ : 1) ao realizar ECR, usando DP e DA nos 50, 100, 110 minutos pós-exercício; 2) posterior à execução do ECR usando DA nos 20, 30, 70, 80,100, 120 minutos; 3) depois de finalizar a sessão ECR com DP nos 10, 40 e 120 minutos. Com relação à medição pré-teste, os valores de PA diminuíram de maneira significativa $(p<0,05)$ nos 10, 20, 40, 50, 60, 100 e 120 minutos pós-ECR usando DP. Em conclusão, ao fazer a comparação com os valores de PA mostrados em um dia de controle, a execução de ECR 
aplicando DP e DA é funcional no controle da PA de homens jovens normotensos. Com relação ao pré-teste, poderia indicar-se que o DP é o promotor do efeito hipotensivo do ECR.

Palavras-chave: descanso ativo, descanso passivo, efeito hipotensor, exercício contrarresistência.

Actualmente, se observa un aumento del número de personas con hipertensión arterial, tanto en países desarrollados como en vías de desarrollo (Hamer, 2006). Por este motivo, y como complemento a la medicación, se han empleado algunas estrategias no farmacológicas que permitan disminuir el impacto de esta enfermedad en la salud pública y en el gasto económico que representa su tratamiento en los diferentes países (Casonatto y Polito, 2009). Una de las estrategias no farmacológicas más recomendada en la literatura es la ejecución de ejercicio (Lemos et al., 2018; Lovato, Anunciação, y Polito, 2012), debido a que se ha demostrado que una sola sesión de entrenamiento puede provocar un efecto hipotensor, es decir, una disminución de la presión arterial (PA), que puede mantenerse por minutos e incluso horas después de finalizada la sesión de ejercicio (Bentes et al., 2017; Della Corte, Paz, de Castro, y Miranda, 2018; Medeiros, Sousa, Lunardi, Oliveira, y Freitas, 2017; Sales et al., 2012).

Sin embargo, a pesar de existir evidencia de que diferentes modalidades de entrenamiento pueden promover un efecto hipotensivo (Carpio-Rivera, Moncada-Jiménez, Salazar-Rojas, y Solera-Herrera, 2016; Corso et al., 2016; Lemos et al., 2018), hay mayor cantidad de investigaciones en los que se reporta este efecto obtenido a partir de una sesión de ejercicio aeróbico (Keese, Farinatti, Pescatello, y Monteiro, 2011). Por lo tanto, es necesario profundizar en el estudio de la hipotensión en otras modalidades de ejercicios, específicamente, el ejercicio contra resistencia (ECR), ya que, en la actualidad, este tipo de entrenamiento se ha popularizado y es muy utilizado en los programas de acondicionamiento físico (Rodríguez, Polito, Bacurau, Prestes, y Pontes, 2008), además de ser un tipo de ejercicio que se prescribe manipulando diversas variables (Lemos et al., 2018), las cuales deben analizarse para determinar un protocolo que permita obtener mayor hipotensión, generando el menor riesgo cardiaco para el participante (Anunciação, Poton, Szytko, y Polito, 2012; Lemos et al., 2018; Veloso et al., 2010).

Una de las variables del ECR que se recomienda analizar son los descansos y, de esta variable, algunas modificaciones que pueden emplearse, como la duración (Lemos et al., 2018) y el tipo de descanso (Brito, Alves, Araújo, Gonçalves, y Silva, 2011). Con respecto al tipo de descanso, se ha hecho referencia a dos modalidades (Brito et al., 2011): primero, el descanso pasivo, en donde la persona permanece en reposo, ya sea sentada o de pie; el segundo es el descanso activo, en el que el individuo puede desplazarse caminando, trotando o corriendo, o bien, ejecutar algunos otros movimientos, por ejemplo, subir y bajar de un step o cajón. La inclusión de los descansos activos entre series y ejercicios ha demostrado incrementar el efecto hipotensor del ECR en adultos mayores hipertensos (Brito et al., 2011), pero es un tipo de descanso que aún no se ha incluido como parte de la investigación en población joven y sana. Por tanto, y con base en la evidencia previa, se desarrolló el presente estudio, el cual tuvo como 
objetivo comparar el efecto del tipo de descanso al realizar ECR sobre la PA de participantes normotensos jóvenes.

\section{MÉTODO}

\section{Participantes}

Participaron en el estudio 9 hombres, con edad promedio de $25.33 \pm 4.53$ años, peso corporal de $75.14 \pm 11.76 \mathrm{~kg}$ y estatura de $1.77 \pm 0.03$ metros, quienes al momento de la investigación no tenían experiencia en ECR. Todos los participantes mantenían valores normales de PA, según los criterios del Octavo Comité Nacional para la prevención, detección, evaluación y tratamiento de la PA (JNC-8 por sus siglas en inglés) (James et al., 2014). No hubo muerte experimental, es decir, el estudio finalizó con la misma cantidad de participantes con que se inició.

La participación fue voluntaria y los individuos firmaron la fórmula de Consentimiento Informado. El estudio fue aprobado por el Comité de Ética de la Universidad Federal de Sergipe. Se cumplieron los principios de la Declaración de Helsinki, respecto a los principios éticos para investigaciones en seres humanos.

Además, se considera relevante indicar que se realizó un cálculo de potencia o poder estadístico a posteriori. Este análisis evidenció que, realizando el estudio con una diferencia mínima a detectar de $8.74 \mathrm{mmHg}$ en la Presión arterial sistólica (PAs), utilizando un nivel de significancia de .01 , con un tamaño de muestra de 9 personas y una variabilidad de $2.22 \mathrm{mmHg}$ en la desviación estándar, se alcanzó una potencia estadística de $90 \%$, valor que se ubica dentro de los porcentajes recomendados (Keppel y Wickens, 2004).

\section{Instrumentos de medición}

Para medir la PA se utilizó un medidor automático marca Microlife ${ }^{\circledR}$ (BP 3AC1-1; Microlife, Switzerland), previamente validado y utilizado en población brasileña (Mota, de Oliveira, Dutra et al., 2013; Mota, Oliveira, Terra et al.,_2013). Para medir el peso corporal y la estatura se utilizó una balanza y un tallímetro marca Filizola ${ }^{\circledR}$. Además, se utilizó un monitor de Frecuencia Cardíaca (FC) marca Polar® (RS100, modelo \#190030907) para controlar la FC durante el periodo de calentamiento y para garantizar que la FC de trabajo de los participantes se

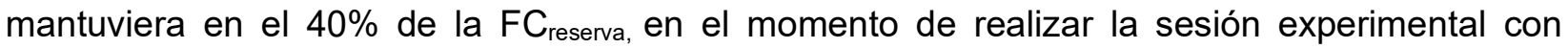
descanso activo (DA).

\section{Procedimientos}

Antes de iniciar las sesiones experimentales, se ejecutaron tres sesiones de familiarización, en las que se realizaron los mismos 10 ejercicios que se emplearon en las sesiones experimentales en las que se debía ejecutar ECR. Estos ejercicios fueron: aducción y abducción de cadera, extensión y flexión de rodilla, extensión y flexión de codo, jalón polea por delante, remo con agarre neutro, press de banca inclinado en máquina y prensa de pierna. Las sesiones de familiarización se realizaron sin carga y con el objetivo de promover en los participantes cierta coordinación neuromuscular. Además, en estas sesiones cada participante recibió la asesoría de la prescriptora del ejercicio a cargo del estudio. Una semana después del proceso de familiarización, se ejecutó el test de $1 \mathrm{RM}$ en los 10 ejercicios previamente mencionados, 
siguiendo el protocolo descrito en literatura previa (Anunciação et al., 2012) y se midieron el peso y la estatura. También se calculó la FC de trabajo que se estableció en $40 \%$ de la FC reserva (ACSM, $\underline{2013}$.

Una semana después, los participantes ejecutaron las tres condiciones experimentales, con un intervalo de tiempo de 72 horas entre sesiones y siguiendo un diseño de investigación de medidas repetidas con orden aleatorizado. Las sesiones experimentales fueron:

1. Descanso activo (DA). Realizar 3 series, 10 repeticiones, con un tempo de 2-2; es decir, 2 tiempos en la fase concéntrica y 2 tiempos en la fase excéntrica, al $70 \%$ de $1 \mathrm{RM}$, con un minuto de DA entre series y ejercicios, en cada uno de los siguientes 10 ejercicios: aducción y abducción de cadera, extensión y flexión de rodilla, extensión y flexión de codo, jalón polea por delante, remo con agarre neutro, press de banca inclinado en máquina y prensa de pierna. EI DA consistió en desplazarse caminando por la sala, manteniendo la

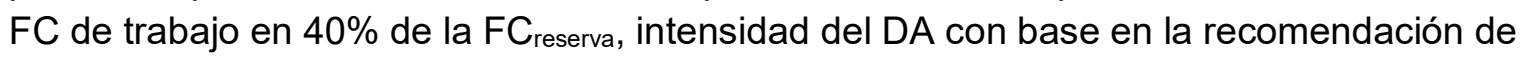
Brito et al. (2011).

2. Descanso pasivo (DP). Realizar 3 series, 10 repeticiones, con un tempo de $2-2$, al $70 \%$ de $1 \mathrm{RM}$, con un minuto de DP entre series y ejercicios, en cada uno de los 10 ejercicios ya indicados previamente. El DP consistió en permanecer sentado en la máquina del ejercicio que se estaba ejecutando.

3. Sesión Control (sin ejercicio). En esta sesión, los participantes permanecieron en reposo (sentados) durante 60 minutos.

Las sesiones con DA y DP estuvieron precedidas por un calentamiento de 5 minutos

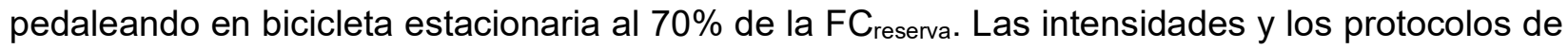
ejercicio se basaron en las recomendaciones brindadas en la guía para la medición y prescripción del ejercicio del Colegio Americano de Medicina Deportiva (ACSM, 2013).

Durante las tres sesiones experimentales (Control, DA y DP), se le solicitó a cada participante evitar la maniobra de Valsalva (exhalar aire mientras se tiene la glotis cerrada) y evitar el consumo de alimentos o bebidas. Asimismo, se les solicitó abstenerse de realizar ejercicio 48 horas antes de cada intervención.

En las tres sesiones experimentales se midió la PA y la FC, siguiendo el protocolo sugerido en literatura previa (Aragón-Vargas y Fernández-Ramírez, 1995). Estas mediciones se realizaron:

a) Pre-tratamiento (reposo). Realizada previo al inicio de la sesión experimental, después de reposar sentado por un período de 10 minutos.

b) Post-tratamiento. Realizada inmediatamente un minuto después de finalizada la sesión experimental y por un período de 120 minutos posteriores al ejercicio, con mediciones cada 10 minutos. En estas mediciones los participantes permanecieron sentados, y desde esta posición se realizaron las mediciones.

\section{Análisis estadístico}

Para la presión arterial sistólica (PAs), presión arterial diastólica (PAd) y frecuencia cardíaca (FC), se calcularon promedios y desviaciones estándar en la estadística descriptiva; además, se 
aplicaron ANOVAS de dos vías para medidas repetidas ( 3 condiciones $\times 14$ mediciones) en la estadística inferencial. Cuando fue necesario, se aplicaron los cálculos de efectos simples y análisis post hoc de Tukey. Se aceptó significancia estadística con $p<.05$. El análisis se realizó por medio del uso del Paquete Estadístico para las Ciencias Sociales SPSS (Chicago, Illinois, USA) versión 21.0 para Windows.

\section{RESULTADOS}

En la Tabla 1 y la Tabla $\underline{2}$ se presenta el resumen de la estadística descriptiva de la PAs, PAd y FC. 
Tabla 1

Resultados de la estadística descriptiva para PAs y PAd en cada condición experimental

\begin{tabular}{|c|c|c|c|c|c|c|}
\hline \multirow[b]{2}{*}{ Medición } & \multicolumn{3}{|c|}{ PAs (mmHg) } & \multicolumn{3}{|c|}{ PAd (mmHg) } \\
\hline & Control & DP & DA & Control & DP & DA \\
\hline Pre-test & $113.89 \pm 7.20$ & $116.67 \pm 12.50$ & $112.22 \pm 12.91$ & $68.89 \pm 7.62$ & $69.33 \pm 9.31$ & $67.78 \pm 9.05$ \\
\hline $1 \mathrm{~min} P E$ & $115.22 \pm 9.05$ & $112.89 \pm 10.69$ & $120.33 \pm 19.03$ & $66.89 \pm 8.02$ & $60.44 \pm 7.38$ & $69.78 \pm 9.35$ \\
\hline $10 \min \mathrm{PE}$ & $111.00 \pm 7.94$ & $106.33 \pm 9.68$ & $107.00 \pm 12.48$ & $68.00 \pm 7.38$ & $60.22 \pm 5.38$ & $61.89 \pm 5.46$ \\
\hline $20 \min P E$ & $112.11 \pm 9.40$ & $106.00 \pm 8.14$ & $108.00 \pm 10.64$ & $69.22 \pm 8.54$ & $63.56 \pm 5.46$ & $63.00 \pm 5.72$ \\
\hline $30 \min P E$ & $113.78 \pm 5.61$ & $108.22 \pm 11.61$ & $105.67 \pm 8.79$ & $68.89 \pm 6.58$ & $64.33 \pm 7.48$ & $62.56 \pm 9.11$ \\
\hline $40 \min P E$ & $112.89 \pm 6.84$ & $107.56 \pm 10.96$ & $104.89 \pm 11.65$ & $69.44 \pm 8.11$ & $63.11 \pm 5.09$ & $64.33 \pm 6.61$ \\
\hline $50 \min P E$ & $112.00 \pm 6.84$ & $105.22 \pm 8.79$ & $103.89 \pm 7.87$ & $67.44 \pm 6.78$ & $61.89 \pm 4.51$ & $63.89 \pm 5.86$ \\
\hline $60 \min \mathrm{PE}$ & $113.00 \pm 8.22$ & 106.6711 .54 & $105.67 \pm 10.02$ & $67.89 \pm 6.23$ & $66.56 \pm 3.24$ & $65.56 \pm 5.39$ \\
\hline $70 \min P E$ & $115.22 \pm 6.46$ & $110.00 \pm 9.80$ & $107.00 \pm 10.58$ & $69.33 \pm 7.21$ & $65.89 \pm 5.95$ & $64.33 \pm 6.22$ \\
\hline $80 \min P E$ & $114.33 \pm 11.01$ & $108.89 \pm 9.18$ & $106.56 \pm 11.91$ & $71.11 \pm 10.34$ & $65.56 \pm 6.75$ & $63.67 \pm 6.60$ \\
\hline $90 \min P E$ & $113.22 \pm 10.60$ & $110.56 \pm 12.65$ & $109.44 \pm 11.51$ & $68.67 \pm 5.24$ & $66.11 \pm 5.44$ & $63.89 \pm 8.51$ \\
\hline 100 min PE & $118.67 \pm 7.62$ & $105.89 \pm 7.47$ & $108.89 \pm 9.41$ & $71.33 \pm 5.92$ & $65.89 \pm 6.33$ & $64.56 \pm 7.70$ \\
\hline 110 min PE & $116.89 \pm 8.52$ & $108.44 \pm 11.15$ & $108.11 \pm 10.49$ & $69.44 \pm 4.10$ & $69.56 \pm 4.50$ & $66.33 \pm 7.97$ \\
\hline $120 \min P E$ & $114.11 \pm 5.11$ & $105.78 \pm 7.92$ & $112.33 \pm 11.26$ & $72.22 \pm 6.46$ & $66.89 \pm 5.40$ & $66.56 \pm 6.64$ \\
\hline
\end{tabular}

Nota. PAs: presión arterial sistólica; PAd: presión arterial diastólica; DP: descanso pasivo; DA: descanso activo; PE: posterior al ejercicio. Fuente: elaboración propia. 
Tabla 2

Resultados de la estadística descriptiva para la FC en cada condición experimental

\begin{tabular}{|c|c|c|c|}
\hline \multicolumn{4}{|c|}{ FC (latidos por minuto) } \\
\hline Medición & Control & DP & DA \\
\hline Pre-test & $67.89 \pm 17.05$ & $68.00 \pm 7.55$ & $68.44 \pm 13.61$ \\
\hline $1 \mathrm{~min} P E$ & $64.89 \pm 16.43$ & $99.33 \pm 26.24$ & $106.33 \pm 27.35$ \\
\hline $10 \min \mathrm{PE}$ & $67.89 \pm 16.89$ & $92.00 \pm 22.79$ & $100.11 \pm 23.98$ \\
\hline $20 \min P E$ & $67.00 \pm 15.68$ & $88.22 \pm 21.22$ & $88.67 \pm 21.78$ \\
\hline $30 \min P E$ & $68.11 \pm 19.25$ & $86.89 \pm 18.66$ & $88.44 \pm 20.83$ \\
\hline $40 \min P E$ & $64.44 \pm 14.38$ & $98.67 \pm 38.31$ & $87.44 \pm 19.91$ \\
\hline $50 \min P E$ & $65.78 \pm 15.84$ & $95.11 \pm 36.70$ & $84.44 \pm 19.88$ \\
\hline $60 \min \mathrm{PE}$ & $64.56 \pm 14.19$ & $82.33 \pm 17.16$ & $83.11 \pm 18.63$ \\
\hline 70 min PE & $63.44 \pm 14.55$ & $78.22 \pm 18.69$ & $82.44 \pm 17.96$ \\
\hline $80 \min P E$ & $63.33 \pm 14.04$ & $77.22 \pm 16.57$ & $78.11 \pm 17.98$ \\
\hline $90 \min \mathrm{PE}$ & $61.67 \pm 11.59$ & $77.22 \pm 17.80$ & $77.56 \pm 18.01$ \\
\hline 100 min PE & $61.67 \pm 13.30$ & $79.56 \pm 16.48$ & $77.22 \pm 20.05$ \\
\hline $110 \min P E$ & $65.22 \pm 13.73$ & $76.00 \pm 17.98$ & $78.67 \pm 18.31$ \\
\hline $120 \min P E$ & $65.33 \pm 14.27$ & $77.89 \pm 16.97$ & $76.00 \pm 18.17$ \\
\hline
\end{tabular}

Nota. FC: frecuencia cardiaca; DP: descanso pasivo; DA: descanso activo; PE: posterior al ejercicio. Fuente: elaboración propia.

\section{Presión arterial sistólica (PAs)}

Se detectó una interacción significativa entre el tiempo de medición y la condición experimental $(F=1.65 ; p=.03)$. Los análisis de efectos simples evidenciaron cambios significativos entre condiciones experimentales a los $50(F=4.55 ; p<.05), 70(F=4.43 ; p$ $<.05), 100(F=10.73 ; p<.05), 110(F=5.95 ; p<.05)$ y 120 minutos $(F=4.62 ; p<.05)$ post ejercicio. Al dar seguimiento a los resultados obtenidos del análisis de efectos simples, se aplicaron análisis post hoc de Tukey que demostraron que, en comparación con los datos obtenidos en la sesión control: 1) la PAs fue significativamente menor en las mediciones realizadas a los 50, 100 y 110 minutos después de haber ejecutado ECR aplicando DA y DP ( $p<.05) ; 2)$ la PAs fue significativamente menor en la medición realizada 70 minutos después de finalizada la sesión de ECR aplicando DA $(p<.05)$; 3$)$ la PAs se mantuvo más baja, de forma significativa, en la medición realizada a los 120 minutos posterior a haber finalizado la sesión de ECR con DP $(p<.05)$ (Figura 1).

Con respecto a comparaciones entre las condiciones experimentales DP y DA, los análisis post hoc de Tukey evidenciaron que: 1) la disminución de la PAs obtenida post ejercicio a los 50, 70, 100, 110 y 120 minutos fue semejante al realizar ECR aplicando DP 
respecto a ejecutar ECR con DA $(p>.05)$. Por otra parte, los análisis de efectos simples demostraron diferencias entre mediciones en la condición experimental DP $(F=4.24 ; p<$ .001). Los análisis post hoc de Tukey evidenciaron que, en comparación con la medición pre-test, la PAs disminuyó de forma significativa $(p<.05)$ a los 10, 20, 50, 60, 100 y 120 minutos después de finalizada la sesión de ECR aplicando DP (ver Figura 1).

\section{Presión arterial diastólica (PAd)}

Se detectó una interacción significativa entre el tiempo de medición y la condición experimental $(F=1.95 ; p=.006)$. Los análisis de efectos simples evidenciaron cambios significativos entre condiciones experimentales en las mediciones de 1 minuto $(F=11.34$; $p<.05), 10$ ( $F=8.32 ; p<.05), 20$ ( $F=5.87 ; p<.05), 30$ ( $F=5.29 ; p<.05), 40$ ( $F=6.40 ; p$ $<.05), 80(F=7.42 ; p<.05), 100(F=6.38 ; p<.05)$ y 120 minutos $(F=5.01 ; p<.05)$ post ejercicio.

Al dar seguimiento a los resultados obtenidos del análisis de efectos simples, se aplicaron análisis post hoc de Tukey los cuales demostraron que, en comparación con los datos obtenidos en la sesión control: 1) la PAd fue significativamente menor en las mediciones realizadas a los 10 y 40 minutos después de haber ejecutado ECR aplicando DP $(p<.05), 2)$ la PAd fue significativamente menor en las mediciones realizadas a los 20 , $30,80,100$ y 120 minutos después de finalizada la sesión de ECR aplicando DA $(p<.05)$ (ver Figura 1).

Con respecto a comparaciones entre las condiciones experimentales DP y DA, los análisis post hoc de Tukey evidenciaron que: 1) la PAd fue significativamente menor al minuto después de finalizada la sesión de ECR con DP en comparación a cuando se realizó la sesión con DA $(p<.05)$, 2) La disminución de la PAd obtenida post ejercicio, fue semejante al realizar ECR aplicando DP respecto a ejecutar ECR con DA en las mediciones realizadas a los $10,20,30,40,80,100$ y 120 minutos después de finalizada la sesión experimental $(p>.05)$. Por otra parte, los análisis de efectos simples demostraron diferencias entre mediciones en la condición experimental DP $(F=4.12 ; p<.01)$. Los análisis post hoc de Tukey evidenciaron que, en comparación con la medición pre-test, la PAd disminuye de forma significativa al minuto y a los 10, 40 y 50 minutos después de finalizada la sesión de ECR aplicando DP $(p<0.05)$ (ver Figura 1). 


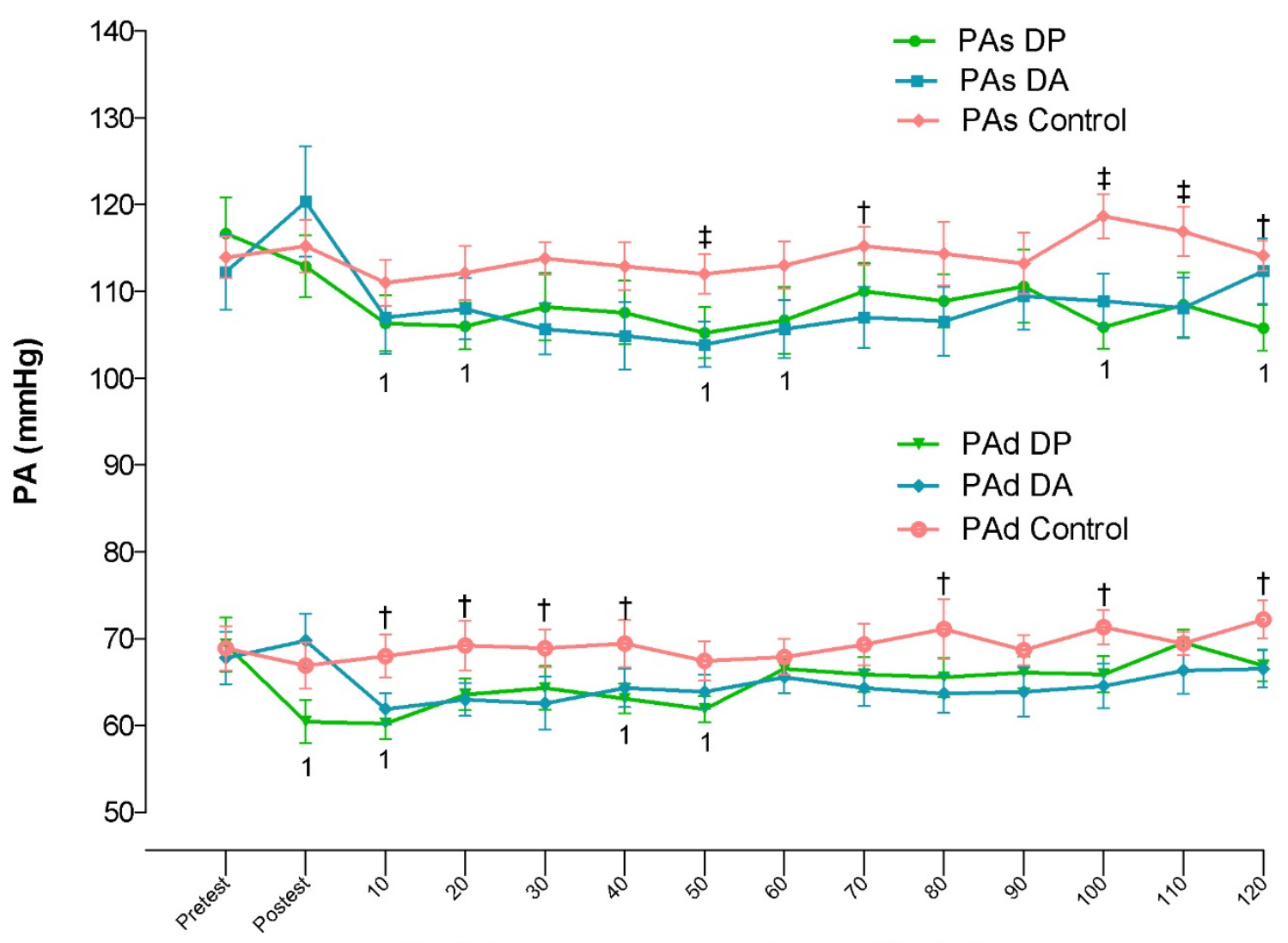

Mediciones pre y post-intervención ( $\mathrm{min})$

Figura 1. Comportamiento de la presión arterial según condición experimental ejecutada. Nota. PAs: presión arterial sistólica; DP: descanso pasivo; DA: descanso activo; PAd: presión arterial diastólica; min: minutos; 1 : diferencia significativa con respecto a la medición pre-test; $\uparrow$ : diferencia significativa entre la condición con PA menor y la condición con PA mayor; $\ddagger$ : diferencia significativa entre las dos condiciones con PA menor y la condición control. Fuente: elaboración propia.

\section{Frecuencia Cardiaca (FC)}

Se detectó una interacción significativa entre el tiempo de medición y la condición experimental $(F=3.80 ; p<.001)$. Los análisis de efectos simples evidenciaron cambios significativos entre condiciones experimentales en las mediciones de 1 minuto $(F=53.84$; $p<.01), 10(F=8.32 ; p<.01), 20(F=5.87 ; p<.01), 30(F=5.29 ; p<.01), 40(F=6.40 ;$ $p<.01), 50(F=7.42 ; p<.01), 60(F=6.38 ; p<.01), 70(F=6.38 ; p<.01), 80(F=6.38 ; p$ $<.01), 90(F=6.38 ; p<.01), 100(F=6.38 ; p<.01), 110(F=6.38 ; p<.01)$ y 120 minutos $(F=5.01 ; p<.01)$ post ejercicio. Al dar seguimiento a los resultados obtenidos del análisis de efectos simples, se aplicaron análisis post hoc de Tukey que demostraron que, en comparación con los datos obtenidos en la sesión control: 1) la FC incrementó de forma 
significativa al minuto y hasta los 120 minutos después de finalizadas las sesiones de ECR aplicando DP y DA (todos los cálculos con $p<.05$ ) (ver Figura 2).

Respecto a comparaciones entre las condiciones experimentales DP y DA, los análisis post hoc de Tukey evidenciaron que: 1) no hubo diferencia significativa en el incremento de la FC al comparar la condición experimental, en la que se incluyó DP, respecto a la condición experimental, en la que se incluyó DA $(p>.05)$. Por otra parte, los análisis de efectos simples demostraron diferencias entre mediciones en la condición experimental DP $(F=9.84 ; p<.01)$ y DA $(F=10.70 ; p<.01)$.

Los análisis post hoc de Tukey evidenciaron que, en comparación con la medición pre-test: 1) la FC se mantuvo aumentada de forma significativa desde el primer minuto post ejercicio y hasta la medición realizada a los 60 minutos después de haber finalizado la sesión de ECR con DP (todos los cálculos con $p<.05$ ), 2) la FC se mantuvo aumentada de forma significativa desde el primer minuto post ejercicio y hasta la medición realizada a los 70 minutos después de haber finalizado la sesión de ECR con DA (todos los cálculos con $p<.05), 3$ ) después del minuto 60 y posterior al minuto 70 de las sesiones de ECR con DP y DA, respectivamente, los valores de FC volvieron a ser semejantes a los encontrados en el pre-test (todos los cálculos con $p>.05$ ).

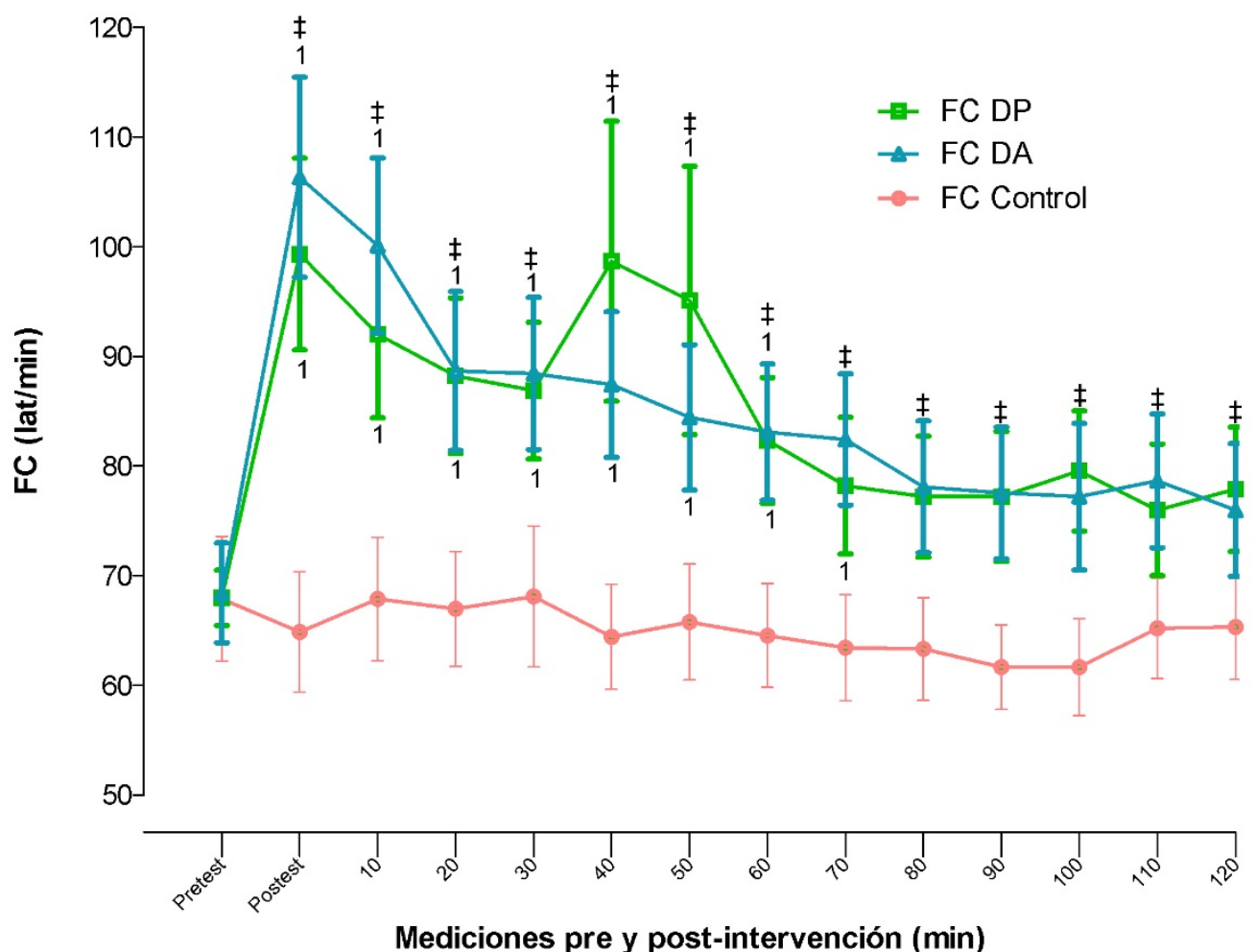

Figura 2. Comportamiento de la frecuencia cardíaca según condición experimental ejecutada. Nota. FC: frecuencia cardíaca; DP: descanso pasivo; DA: descanso activo; min: minutos; 1: diferencia significativa con respecto a la medición pre-test; $\ddagger$ : diferencia 
significativa entre las dos condiciones con FC mayor y la condición control. Fuente: elaboración propia.

\section{DISCUSIÓN}

El estudio tuvo como objetivo comparar el efecto del tipo de descanso al realizar ECR, sobre la PA de participantes normotensos. Uno de los resultados más importantes es demostrar que, en relación a un día de control (sin ejercicio), los valores de PA disminuyeron de forma significativa al realizar ECR utilizando DP y DA a los 50, 100, 110 minutos postejercicio; posterior a ejecutar ECR utilizando DA a los 20, 30, 70, 80,100, 120 minutos; y después de finalizada la sesión ECR, con DP a los 10, 40 y 120 minutos. Asimismo, se demostró que, en relación con la medición pre-test, los valores de PA disminuyeron de forma significativa a los 10, 20, 40, 50, 60, 100 y 120 minutos post ECR utilizando DP.

En este punto, es prudente aclarar que, metodológicamente, existen dos formas posibles para evaluar el efecto hipotensor del ejercicio: 1) comparando los datos de PA obtenidos en un día en que se realice ejercicio con los valores de PA obtenidos en un día de control (sin ejercicio), y 2) comparar los valores de PA del pre-test con los datos de PA obtenidos en las mediciones post-sesión de entrenamiento (Baum, Rüther, y Essfeld, 2003). Tomando esto en consideración, el presente estudio demostró un efecto hipotensor del ECR aplicando ambas formas metodológicas mencionadas.

Ahora bien, sin querer minimizar la importancia del resultado que demostró que, respecto al pre-test, se obtiene un efecto hipotensor únicamente cuando se realiza ECR con DP, se considera oportuno destacar el hallazgo que mostró que, respecto a un día de control, realizar ECR aplicando DP o DA genera un efecto hipotensivo. Esta información evidencia la importancia de este tipo de ejercicio, independientemente del modo de descanso utilizado, como una estrategia no farmacológica posible para el control de la PA en los participantes que constituyeron la muestra de la presente investigación.

Con respecto a posibles comparaciones entre estudios, se podría indicar que los resultados aquí expuestos concuerdan con la evidencia previa. Por ejemplo, en investigaciones experimentales y un meta-análisis, en los que analizaron población semejante a la incluida en el presente estudio (Bentes et al., 2017; Gamboa-Granados y Solera-Herrera, 2017; Quesada-Valle, Carpio-Rivera, y Solera-Herrera, 2018; Quesada Valle y Solera-Herrera, 2017), demostraron una disminución significativa de la PA después de finalizada la sesión de ECR, ejecutada de forma tradicional, es decir, aplicando DP.

Ahora bien, el resultado que evidenció que el uso de DA no incrementó el efecto hipotensivo del ECR, difiere de lo demostrado en el único estudio ubicado (Brito et al., 2011), en el que se evaluó de forma específica la influencia del tipo de descanso (DA, DP) sobre la PA. La diferencia entre los resultados demostrados por ambas investigaciones podría deberse al tipo de población analizada, ya que mientras en el estudio de Brito et al. (2011) se evaluaron mujeres adultas mayores hipertensas, en el presente estudio se analizaron hombres jóvenes y sanos. Literatura previa (Canuto et al., 2011), ha mencionado que, con la edad, se incrementa la rigidez arterial, el grueso de las arteriolas y se disminuye la capacidad funcional del endotelio. Por tanto, se ha expresado que en el caso de personas

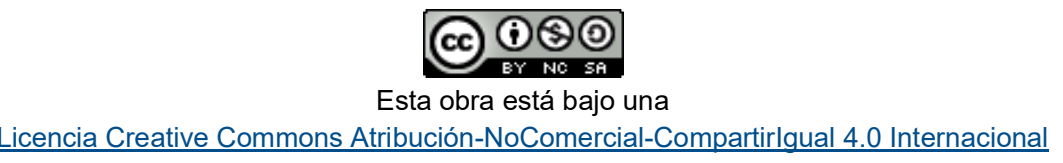



Ejercicio contra resistencia y Presión Arterial

adultas mayores se torna necesario la inclusión de DA en las sesiones de ECR, de manera que se promueva un aumento del flujo sanguíneo y, con este, un aumento del mecanismo de cizallamiento sobre las paredes de las células endoteliales, mecanismo, que según algunos autores (Brito et al., 2011), es necesario para que se dé la liberación de sustancias vasodilatadoras. Al respecto, se torna interesante, para estudios futuros, comparar el efecto de diferentes tipos de descanso sobre la PA de personas con edades diferentes, para determinar si la respuesta es distinta dependiendo de la edad del participante.

Otra posible explicación a la diferencia entre los resultados evidenciados por Brito et al. (2011) y los hallazgos mostrados en el presente estudio, podría ser el hecho de que la

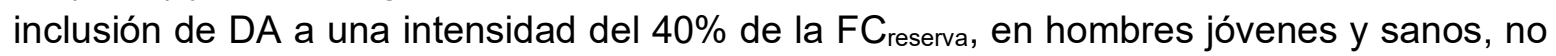
representó un estímulo suficiente para incrementar el efecto hipotensivo. Este argumento se respalda en los hallazgos expuestos por otros autores (Medeiros et al., 2017), quienes demostraron un efecto hipotensivo después de que hombres jóvenes normotensos realizaron una sesión de ejercicio concurrente en el que ejecutaban el ECR al $50 \%$ de $1 \mathrm{RM}$ y el ejercicio aeróbico en un intervalo de entre $80-90 \%$ de la $\mathrm{FC}_{\text {reserva. }}$. Por esta razón, se recomienda desarrollar investigaciones al respecto. Esta recomendación se fundamenta, además, en la necesidad detectada por otros autores (Lamberti et al., 2016), quienes sugieren desarrollar estudios aplicando ejercicio concurrente, el cual, al combinar los beneficios del ejercicio aeróbico y ECR en una misma sesión, puede promover un efecto hipotensivo con valores clínicamente relevantes.

Ahora bien, en la literatura previa se ha mencionado que, aún los mecanismos fisiológicos que podrían explicar el efecto hipotensivo generado a partir de una sesión de ECR no están completamente claros (del Valle Soto et al., 2015; Queiroz, Kanegusuku, y Forjaz, 2010). A pesar de ello, algunos de los estudios en los que han evaluado posibles mecanismos fisiológicos han explicado que la hipotensión lograda a partir de una sesión de ECR puede deberse a: 1) modificaciones del gasto cardíaco (GC) y la resistencia vascular periférica total (Brito, Oliveira, Santos, y Santos, 2014; Moraga Rojas, 2008), 2) modificaciones neurohumorales (Figueiredo et al., 2015; Queiroz et al., 2015; Rezk, Marrache, Tinucci, Mion, y Forjaz, 2006; Teixeira, Ritti-Dias, Tinucci, Júnior, y de Moraes Forjaz, 2011), 3) incremento en sustancias vasodilatadoras como el óxido nítrico (Bentes et al., 2015) y las cininas (Moraes et al., 2007).

Con respecto a las investigaciones que han evaluado la relación entre la disminución de la PA con la modificación del GC o resistencia vascular sistémica, han demostrado reducción de alguna de estas variables después de la sesión de ECR (Brito, Oliveira, Santos, y Santos, 2014; Queiroz et al., 2013; Rezk et al., 2006; Teixeira et al., 2011) o modificación no significativa de ellas (Queiroz et al., 2015). Estas controversias, con respecto a las respuestas hemodinámicas que provocan el efecto hipotensivo (Queiroz et al., 2015), demuestran que es un tema que requiere más investigación, ya que incluso se ha demostrado que no todos los individuos responden de manera semejante (Casonatto, Goessler, Cornelissen, Cardoso, y Polito, 2016; Queiroz et al., 2015).

En relación con el mecanismo que relaciona la hipotensión post ECR con sustancias vasodilatadoras, se ha demostrado que son el óxido nítrico y las cininas las sustancias que, 
al incrementarse en plasma, permiten mayor vasodilatación y por ende disminución en la PA (Lizardo, Silveira, Vassallo, y Oliveira, 2008; Moraes et al., 2007). Se ha descrito que el factor principal que produce la liberación de estas sustancias es el cizallamiento o roce de la sangre sobre la capa de las células endoteliales, el cual se acentúa por el aumento del flujo sanguíneo durante el ejercicio (de Freitas Brandão y Pinge, 2007; Lizardo et al., 2008; Moraes et al., 2007).

En este punto, se considera importante mencionar que es poca la cantidad de estudios que han evaluado las respuestas hemodinámicas y autonómicas cardiovasculares al ECR. De la misma forma, es escasa la información respecto al comportamiento de las sustancias vasodilatadoras después de una sesión de entrenamiento con pesas, por lo que se considera pertinente desarrollar investigaciones en las que se analicen estos cuestionamientos, para aclarar la contribución de estos mecanismos en el efecto hipotensor logrado por el ECR, ya que como se ha mencionado en la literatura previa, estos mecanismos siguen siendo controvertidos y requieren de mayor investigación (Queiroz et al., 2015).

De esta manera, se reconoce como limitación del estudio no evaluar mecanismos fisiológicos posibles que contribuyeran a explicar el efecto hipotensivo obtenido a partir de las sesiones de ECR aplicando DA y DP. Por otra parte, se reconoce como otra limitación importante, medir la PA por tan solo 120 minutos, lo que limita la capacidad de evaluar por cuánto tiempo se mantuvo la hipotensión evidenciada. Para futuras investigaciones, se recomienda realizar la medición ambulatoria de la PA, la cual permite recolectar los valores de presión sanguínea por un período de 24 horas, con lo que se conoce de manera más precisa el comportamiento de la PA durante actividades cotidianas y durante el período de sueño.

En cuanto a la FC, se demostró que durante los 120 minutos de seguimiento postejercicio, fue más alta al realizar ECR con DP y con DA, en comparación con el reposo o el día control, sin que este aumento fuera distinto según el tipo de descanso que se aplicara en la sesión de entrenamiento. Este aumento de la FC posterior a las sesiones de ECR con DP y con DA fue el comportamiento esperado y puede explicarse por el aumento de la actividad del barorreflejo, el cual responde incrementando la FC cuando detecta disminución de la PA, respuesta que se da con el objetivo de mantener la PA en valores que la persona muestra continuamente (Anunciação et al., 2012).

Por tanto y con base en los resultados previamente expuestos, se concluye que en relación con el día control: 1) en sujetos jóvenes normotensos, la inclusión de DA con

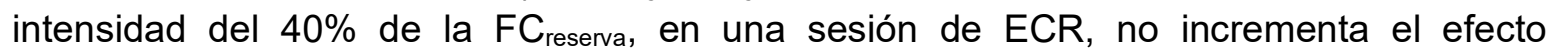
hipotensivo; 2) en esta población, ambos tipos de descanso (DP y DA) son funcionales para el control de la PA, debido a que ambos permiten una disminución significativa de la presión sanguínea posterior a la sesión de entrenamiento. En relación con el pre-test, se puede indicar que, en la población analizada, es el DP el que promueve un efecto hipotensivo, tanto en la PAs como en la PAd. 


\section{REFERENCIAS}

American College of Sports Medicine (ACSM). (2013). ACSM's guidelines for exercise testing and prescription. Estados Unidos: Lippincott Williams \& Wilkins.

Anunciação, P. G., Poton, R., Szytko, A., y Polito, M. D. (2012). Comportamento cardiovascular após o exercício resistido realizado de diferentes formas e volumes de trabalho. Revista Brasileira de Medicina do Esporte, 18(2), 117-121. doi: https://doi.org/10.1590/S1517-86922012000200011

Aragón-Vargas, L., y Fernández-Ramírez, A. (1995). Fisiología del ejercicio: respuestas, entrenamiento y medición. San José: Universidad de Costa Rica.

Baum, K., Rüther, T., y Essfeld, D. (2003). Reduction of blood pressure response during strength training through intermittent muscle relaxations. International Journal of Sports Medicine, 24(6), 441-445. doi: https://doi.org/10.1055/s-2003-41172

Bentes, C. M., Costa, P. B., Corrêa, V. G., Simão, R., Paz, G. A., Maia, M. F., ... y Miranda, H. (2017). Hypotensive Responses of Reciprocal Supersets versus Traditional Resistance Training in Apparently Healthy Men. International Journal of Exercise Science, 10(3), 434-445. Recuperado de https://www.ncbi.nlm.nih.gov/pmc/ articles/PMC5421986/

Bentes, C. M., Costa, P. B., Neto, G. R., Costa e Silva, G. V., Salles, B. F., Miranda, H. L., y Novaes, J. S. (2015). Hypotensive effects and performance responses between different resistance training intensities and exercise orders in apparently health women. Clinical Physiology and Functional Imaging, 35(3), 185-190. doi: https://doi.org/10.1111/cpf.12144

Brito, A. F., Alves, N. F., Araújo, A. S., Gonçalves, M. C., y Silva, A. S. (2011). Active intervals between sets of resistance exercises potentiate the magnitude of postexercise hypotension in elderly hypertensive women. The Journal of Strength \& Conditioning Research, 25(11), 3129-3136. doi: https://doi.org/10.1519/ jsc.0b013e318212dd25

Brito, A. F., Oliveira, C. V. C., Santos, M. S. B., y Santos, A. C. (2014). High-intensity exercise promotes postexercise hypotension greater than moderate intensity in elderly hypertensive individuals. Clinical Physiology and Functional Imaging, 34(2), 126-132. doi: https://doi.org/10.1111/cpf.12074

Canuto, P. M. B. C., Nogueira, I. D. B., Cunha, E. S., Ferreira, G. M. H., Mendonça, K. M. P. P., Costa, F. A., y Nogueira, P. A. M. S. (2011). Influence of resistance training performed at different intensities and same work volume in BP of elderly hypertensive female patients. Revista Brasileira de Medicina do Esporte, 17(4), 246-249. Recuperado de https://www.scielo.br/pdf/rbme/v17n4/en v17n4a06.pdf

Carpio-Rivera, E., Moncada-Jiménez, J., Salazar-Rojas, W., y Solera-Herrera, A. (2016). Acute Effects of Exercise on Blood Pressure: A Meta-Analytic Investigation. Arquivos Brasileiros de Cardiologia 106(5), 422-433. doi: http://dx.doi.org/10.5935/ abc. 20160064 

Ejercicio contra resistencia y Presión Arterial

Casonatto, J., Goessler, K. F., Cornelissen, V. A., Cardoso, J. R., y Polito, M. D. (2016). The blood pressure-lowering effect of a single bout of resistance exercise: a systematic review and meta-analysis of randomised controlled trials. European Journal of Preventive Cardiology, 23(16), 1700-1714. doi: https://doi.org/10.1177/2047487 316664147

Casonatto, J., y Polito, M. D. (2009). Hipotensão Pós-exercício Aeróbio: Uma Revisão Sistemática. Revista Brasileira de Medicina do Esporte, 15(2), 151-157. Recuperado de https://www.researchgate.net/publication/262543128 Post-exercisehypotension a systematic review

Corso, L. M., Macdonald, H. V., Johnson, B. T., Farinatti, P., Livingston, J., Zaleski, A. L., ... Pescatello, L. S. (2016). Is concurrent training efficacious antihypertensive therapy? A meta-analysis. Medicine \& Science in Sports \& Exercise, 48(12), 2398-2406. doi: https://doi.org/10.1249/mss.0000000000001056

Della Corte, J., Paz, G. A., de Castro, J. B. P., y Miranda, H. (2018). Hypotensive effect induced by strength training using the DeLorme and Oxford methods in trained men. Polish Journal of Sport and Tourism, 25(1), 23-30. doi: https://doi.org/10.2478/pjst2018-0004

Figueiredo, T., Rhea, M. R., Peterson, M., Miranda, H., Bentes, C.M., dos Reis, V. M. R., y Simão, R. (2015). Influence of number of sets on blood pressure and heart rate variability after a strength training session. The Journal of Strength \& Conditioning Research, 29(6), 1556-1563. doi: https://doi.org/10.1519/jsc.0000000000000774

de Freitas Brandão, A., y Pinge, M. C. M. (2007). Alteração do óxido nítrico na função cardiovascular pelo treinamento físico. Semina: Ciências Biológicas e da Saúde, 28(1), 53-68. doi: http://dx.doi.org/10.5433/1679-0367.2007v28n1p53

Gamboa-Granados, M., y Solera-Herrera, A. (2017). Efecto agudo de dos intensidades de ejercicio contra resistencia sobre la presión arterial en reposo de personas normotensas. Pensar en Movimiento: Revista de Ciencias del Ejercicio y la Salud, 15(2), e29809. doi: https://doi.org/10.15517/pensarmov.v15i2.29809

Hamer, M. (2006). The anti-hypertensive effects of exercise. Sports Medicine, 36(2), 109116. doi: https://doi.org/10.2165/00007256-200636020-00002

James, P. A., Oparil, S., Carter, B. L., Cushman, W. C., Dennison-Himmelfarb, C., Handler, J., ... Ortiz, E. (2014). 2014 evidence-based guideline for the management of high blood pressure in adults: report from the panel members appointed to the Eighth Joint National Committee (JNC 8). JAMA, 311(5), 507-520. doi: https://doi.org/10.1001/jama.2013.284427

Keese, F., Farinatti, P., Pescatello, L., y Monteiro, W. (2011). A comparison of the immediate effects of resistance, aerobic, and concurrent exercise on postexercise hypotension. The Journal of Strength \& Conditioning Research, 25(5), 1429-1436. doi: https://doi.org/10.1519/jsc.0b013e3181d6d968

Keppel, G., y Wickens, T. D. (2004). Design and Analysis: A Researcher's Handbook. New jersey, Estados Unidos: Pearson Prentice Hall. 

Ejercicio contra resistencia y Presión Arterial

Lemos, S., Figueiredo, T., Marques, S., Leite, T., Cardozo, D., Willardson, J. M., y Simão, R. (2018). Effects of strength training sessions performed with different exercise orders and intervals on blood pressure and heart rate variability. International journal of exercise science, 11(2), 55-67. Recuperado de https://www.ncbi.nlm.nih.gov/pmc/ articles/PMC5955307/

Lizardo, J. H. F., Silveira, E. A. A, Vassallo, D. V., y Oliveira, E. M. (2008). Post-resistance exercise hypotension in spontaneously hypertensive rats is mediated by nitric oxide. Clinical and Experimental Pharmacology and Physiology, 35(7), 782-787. doi: https://doi.org/10.1111/j.1440-1681.2008.04950.x

Lovato, N. S., Anunciação, P. G., y Polito, M. D. (2012). Blood pressure and heart rate variability after aerobic and weight exercises performed in the same session. Revista Brasileira de Medicina do Esporte, 18(1), 22-25. Recuperado de https://www.scielo.br/ pdf/rbme/v18n1/en 04.pdf

Medeiros, A. L. F., Sousa, M. V., Lunardi, M., Oliveira, S. N., y Freitas, C. R. (2017). Effect of a concurrent training session with different intensities in post-exercise blood pressure responses in normotensive adults. Motricidade, 13(4), 33-38. doi: http://dx.doi.org/10.6063/motricidade.11695

Moraes, M. R., Bacurau, R. F., Ramalho, J. D., Reis, F. C., Casarini, D. E., Chagas, J. R., ... Pesquero, J. L. (2007). Increase in kinins on post-exercise hypotension in normotensive and hypertensive volunteers. Biological chemistry, 388(5), 533-540. doi: https://doi.org/10.1515/bc. 2007.055

Moraga Rojas, C. (2008). Prescripción de ejercicio en pacientes con hipertensión arterial. Revista Costarricense de Cardiología, 10(1-2), 19-23. Recuperado de https://www.scielo.sa.cr/pdf/rcc/v10n1-2/3933.pdf

Mota, M. R., de Oliveira, R. J., Dutra, M. T., Pardono, E., Terra, D. F., Lima, R. M., ... da Silva, F. M. (2013). Acute and chronic effects of resistive exercise on blood pressure in hypertensive elderly women. The Journal of Strength \& Conditioning Research, 27(12), 3475-3480. doi: https://doi.org/10.1519/jsc.0b013e31828f2766

Mota, M. R., Oliveira, R. J., Terra, D. F., Pardono, E., Dutra, M. T., de Almeida, J. A., y Silva, F. M. (2013). Acute and chronic effects of resistance exercise on blood pressure in elderly women and the possible influence of ACE I/D polymorphism. International Journal of General Medicine, 6, 581-587. doi: https://doi.org/10.2147/ijgm.s40628

Queiroz, A., Rezk, C., Teixeira, L., Tinucci, T., Mion, D., y Forjaz, C. (2013). Gender influence on post-resistance exercise hypotension and hemodynamics. International Journal of Sports Medicine, 34(11), 939-944. doi: https://doi.org/10.1055/s-0033$\underline{1337948}$

Queiroz, A., Sousa, J., Cavalli, A., Silva, N., Costa, L., Tobaldini, E., ... Forjaz, C. (2015). Post-resistance exercise hemodynamic and autonomic responses: Comparison between normotensive and hypertensive men. Scandinavian Journal of Medicine \& Science in Sports, 25(4), 486-494. doi: https://doi.org/10.1111/sms.12280 

Ejercicio contra resistencia y Presión Arterial

Queiroz, A. C. C., Kanegusuku, H., y Forjaz, C. L. M. (2010). Efectos del Entrenamiento de Resistencia sobre la Presión Arterial de Añosos. Arquivos Brasileiros de Cardiologia, 95(1), 135-140. doi: http://dx.doi.org/10.1590/S0066-782X2010001100020

Quesada-Valle, E., Carpio-Rivera, E., y Solera-Herrera, A. (2018). Influencia del volumen y la carga total del entrenamiento contra resistencia sobre la presión arterial post ejercicio: una investigación meta analítica. Revista Facultad de Ciencias de la Salud UDES, 5(2), 34-43. doi: http://dx.doi.org/10.20320/rfcsudes.v5i2.105

Quesada Valle, E. y Solera-Herrera, A. (2017). Efecto agudo del orden de ejecución de ejercicios contra resistencia sobre la presión arterial en jóvenes estudiantes normotensos. Retos: nuevas tendencias en educación física, deporte y recreación, (32), 68-71. doi: https://doi.org/10.47197/retos.v0i32.49703

Rezk, C., Marrache, R., Tinucci, T., Mion, D., y Forjaz, C. (2006). Post-resistance exercise hypotension, hemodynamics, and heart rate variability: influence of exercise intensity. European Journal of Applied Physiology, 98(1), 105-112. doi: https://doi.org/10.1007/s00421-006-0257-y

Rodríguez, D., Polito, M. D., Bacurau, R. F., Prestes, J., y Pontes, F. (2008). Effect of different resistance exercise methods on post-exercise blood pressure. International Journal of Excercise Science, 1(4), 153-162. Recuperado de https://www.researchgate.net/publication/28330591 Effect of Different Resistance Exercise Methods on Post-Exercise Blood Pressure

Sales, M. M., Russo, P., Moreira, S. R., Santana, H., Moraes, J. F., Asano, R. Y., MotaSantos, D., ... Campbell, C. (2012). Resistance exercise elicits acute blood pressure reduction in type 2 diabetics. Journal Exercise Physiology Online, 15(1), 98-109.

Teixeira, L., Ritti-Dias, R. M., Tinucci, T., Júnior, D. M., y de Moraes Forjaz, C. L. (2011). Post-concurrent exercise hemodynamics and cardiac autonomic modulation. European Journal of Applied Physiology, 111(9), 2069-2078. doi: https://doi.org/10.1007/s00421-010-1811-1

del Valle Soto, M., Manonelles Marqueta, P., de Teresa Galván, C., Franco Bonafonte, L., Luengo Fernández, E., y Gaztañaga Aurrekoetxea, T. (2015). Prescripción de ejercicio físico en la prevención y tratamiento de la hipertensión arterial. Documento de Consenso de la Sociedad Española de Medicina del Deporte (SEMED-FEMEDE). Archivos de Medicina del Deporte, 32(5), 281-312. Recuperado de http://archivosdemedicinadeldeporte.com/articulos/upload/or02 del-valle.pdf

Veloso, J., Polito, M. D., Riera, T., Celes, R., Vidal, J. C., y Bottaro, M. (2010). Effects of Rest Interval between Exercise Sets on Blood Pressure after Resistance Exercises. Arquivos Brasileiros de Cardiologia, 94(4), 512-518. doi: https://doi.org/10.1590/s0066-782x2010005000019

Participación: A- Financiamiento, B- Diseño del estudio, C- Recolección de datos, D- Análisis estadístico e interpretación de resultados, E- Preparación del manuscrito. 